\title{
CARACTERÍSTICAS DE LAS FUNGEMIAS EN UN CENTRO DE REFERENCIA DEL PERÚ: ANÁLISIS RETROSPECTIVO DE CINCO AÑOS
}

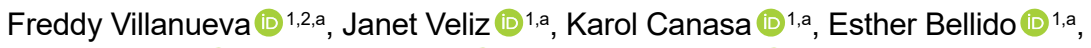 \\ Shirley Martell (iD) ${ }^{1, a}$, Sherly Ortega (iD ${ }^{1, a}$, Ines Cajamarca (iD) 3 ,a \\ ${ }^{1}$ Instituto Nacional de Enfermedades Neoplásicas, Lima, Perú. \\ ${ }^{2}$ Departamento Académico de Microbiología Médica. Universidad Nacional Mayor de San Marcos, Lima, Perú. \\ ${ }^{3}$ Instituto Nacional de Ciencias Neurológicas, Lima, Perú. \\ a Tecnólogo médico.
}

El presente estudio fue presentado por Villanueva F, Veliz J, Canasa K, Bellido E, Martell S, Ortega S, et al. Epidemiología de las fungemias en pacientes oncológicos en un centro de referencia de Perú: una visión retrospectiva de 5 años, en el IV Encuentro Andino de la Asociación Panamericana de Infectología en septiembre de 2018 en Lima, Perú.

\section{RESUMEN}

Con el objetivo de conocer las características de las fungemias en 285 pacientes oncológicos hospitalizados del 2012 al 2016 en el Instituto Nacional de Enfermedades Neoplásicas se realizó un estudio descriptivo retrospectivo. Se evaluó información demográfica, clínica y microbiológica. Las fungemias por C. albicans predominaron en pacientes con tumores sólidos y sin neutropenia, mientras las causadas por C. tropicalis predominaron en pacientes con neoplasias hematológicas y neutropenia. C. tropicalis fue el agente más aislado (47,0\%). Las fungemias aumentaron con el tiempo en los pacientes sin neutropenia. Las fungemias causadas por C. albicans aumentan con la edad en pacientes con tumores sólidos sin neutropenia. Se concluye que las fungemias son mayormente causadas por $C$. tropicalis en pacientes con neoplasias hematológicas con neutropenia y por C. albicans en pacientes con tumores sólidos sin neutropenia. Además, las fungemias en pacientes sin neutropenia aumentan en el tiempo y las causadas por C. albicans, en tumores sólidos sin neutropenia, aumentan con la edad.

Palabras clave: Epidemiología; Tendencias; Etiología; Micosis Invasivas; Candidemia; Hospitales de Cáncer (fuente: DeCS BIREME).

\section{CHARACTERISTICS OF FUNGEMIAS IN A PERUVIAN REFERRAL CENTER: 5-YEAR RETROSPECTIVE ANALYSIS}

Citar como: Villanueva F, Veliz J, Canasa K, Bellido E, Martell S, Ortega S, et al. Características de las fungemias en un centro de referencia del Perú: retrospectiva de cinco años. Rev Peru Med Exp Salud Pública. 2020;37(2):276-81. doi: https://doi. org/10.17843/rpmesp.2020.372.5026

Correspondencia: Freddy Villanueva; Laboratorio de Microbiología, Instituto Nacional de Enfermedades Neoplásicas. Avenida Angamos Este 2520, Lima 34, Perú; fvillanueva@inen. sld.pe

Recibido: $07 / 12 / 2019$

Aprobado: $25 / 03 / 2020$

En línea: $05 / 06 / 2020$

\begin{abstract}
Retrospective descriptive study carried out to determine the characteristics of fungemias in 285 cancer patients hospitalized from 2012 to 2016 at the Instituto Nacional de Enfermedades Neoplásicas (INEN). Demographic, clinical and microbiological information was evaluated. Fungemia by C. albicans predominated in patients with solid tumors and without neutropenia, while those caused by C. tropicalis predominated in patients with hematological neoplasia and neutropenia. C. tropicalis was the agent isolated in most cases (47.0\%). Fungemia increases over time in patients without neutropenia. Fungemia caused by $C$. albicans increases with age in patients with solid tumors without neutropenia. It is concluded that fungemias are mainly caused by $C$. tropicalis in patients with hematological neoplasia with neutropenia and by $C$. albicans in patients with solid tumors without neutropenia. In addition, fungemia in patients without neutropenia increases over time; and those caused by $C$. albicans increase with age in patients with solid tumors without neutropenia.
\end{abstract}

Keywords: Epidemiology; Trends; Etiology; Invasive Mycoses; Candidemia; Cancer Care Facilities (source: MeSH NLM).

\section{INTRODUCCIÓN}

Las fungemias son las micosis invasivas más frecuentes, presentan una alta morbilidad y mortalidad, y se han incrementado debido al aumento de pacientes inmunocomprometidos, sobre todo de los casos oncológicos ${ }^{(1,2)}$. Las fungemias varían según determinadas características demográficas (edad, sexo y región geográfica) y clínicas (enfermedades subyacentes, foco in- 
feccioso primario y estado de neutropenia). Además, la etiología desempeña un papel importante en su aparición ${ }^{(3,4)}$.

La etiología de las fungemias es variada y comprende tanto a hongos filamentosos como a hongos levaduriformes, predominando estos últimos. Aunque Candida albicans (C. albicans) ha sido por mucho tiempo la especie más aislada, otras especies de Candida y otras levaduras diferentes de Candida parecen emerger y desplazar a C. albicans ${ }^{(5,6)}$. Además, estos patógenos presentan diversos perfiles de sensibilidad a los antifúngicos ${ }^{(7)}$. Por lo tanto, la epidemiología y etiología asociadas tienen implicancias en el manejo terapéutico y en el desenlace de las fungemias. Las características demográficas, clínicas y etiológicas de las fungemias han sido poco descritas en nuestra población. Conocer las características de las fungemias a nivel hospitalario permitirá un mejor manejo del control y el tratamiento de estas micosis invasivas.

El objetivo del estudio es determinar las características de las fungemias en pacientes oncológicos hospitalizados desde el 2012 al 2016 en el Instituto Nacional de Enfermedades Neoplásicas (INEN) del Perú.

\section{EL ESTUDIO}

Se realizó un estudio descriptivo retrospectivo de los casos de fungemias diagnosticadas en el laboratorio de microbiología del INEN entre enero del 2012 y diciembre del 2016. La información se obtuvo del registro del laboratorio de Microbiología y del sistema informático del INEN. Se incluyeron todos los casos en los que se pudo identificar el agente etiológico. Los aislamientos duplicados que correspondían a un mismo paciente fueron excluidos del estudio.

El diagnóstico de las fungemias se realizó mediante hemocultivos procesados en el sistema automatizado BD Bactec $^{\mathrm{TM}}$ Fx. La identificación de levaduras se hizo mediante el estudio morfológico, según la técnica de Dalmau en agar almidón arroz, y el bioquímico, según las características cromogénicas en medio CHROMagar y las características de asimilación de carbohidratos en el sistema comercial API 20C AUX system (bioMérieux) ${ }^{\left({ }^{(8)}\right.}$. Los hongos filamentosos fueron identificados mediante sus características macroscópicas y microscópicas ${ }^{(9)}$.

La fungemia se definió como el aislamiento de un hongo en sangre o en médula ósea ${ }^{(10)}$. Adicionalmente, se evaluaron las características demográficas, como la edad (grupo etario) y el sexo; las características clínicas, como el departamento médico, el tipo de neoplasia, el diagnóstico oncológico y el estado de neutropenia definido como el recuento $<1000$ neutrófilos $/ \mathrm{mm}^{3}{ }^{(11)}$. También se evaluaron características microbiológicas, como la etiología (agrupada en C. albicans, Candida no albicans, otras levaduras y filamentosos) y la muestra sembrada.

Se utilizó estadística descriptiva, los resultados se resumieron en frecuencias y porcentajes, y en gráficos de líneas

\section{MENSAJES CLAVE}

Motivación para realizar el estudio: Las fungemias tienen altas tasas de morbilidad y mortalidad en pacientes oncológicos, por lo que resulta necesario conocer sus características hospitalarias.

Principales hallazgos: Las fungemias por C. albicans predominaron en pacientes con tumores sólidos y sin neutropenia, mientras que las causadas por $C$. tropicalis predominaron en pacientes con neoplasias hematológicas y con neutropenia. C. tropicalis fue el agente etiológico más frecuente $(47,0 \%)$. Las fungemias en pacientes sin neutropenia aumentan en el tiempo y las causadas por C. albicans en tumores sólidos sin neutropenia aumentan con la edad.

Implicancias: El conocimiento de las características epidemiológicas en nuestra población mejorará la terapia y el control de estas micosis invasivas.

y barras para evaluar la distribución de las variables. Este estudio se realizó con datos del registro del laboratorio de microbiología y del sistema informático del INEN, por lo que no representa ningún riesgo para los pacientes. El estudio fue evaluado y aprobado por el Comité de Investigación del INEN.

\section{HALLAZGOS}

Durante los cinco años de estudio se realizaron 36923 hemocultivos, de los cuales $285(0,8 \%)$ resultaron ser casos de fungemias, las cuales se incrementaron en el tiempo. En el 2012 se realizaron 5814 hemocultivos, y se encontraron 34 casos (0,7\%); en el 2013 se realizaron 6400 hemocultivos, y se encontraron 41 casos (0,6\%); en el 2014 se realizaron 7438 hemocultivos, y se encontraron 59 casos (0,8\%); en el 2015 se realizaron 7861 hemocultivos, y se encontraron 71 casos $(0,9 \%)$; finalmente en el 2016 se realizaron 9410 hemocultivos, y 80 casos $(0,9 \%)$ resultaron ser fungemias.

Fueron más frecuentes el grupo etario de 0 a 19 años $(27,4 \%)$ y el sexo femenino $(54,4 \%)$. El $56,5 \%$ de los pacientes tuvieron neutropenia; el 64,6\%, neoplasias hematológicas; el $31,9 \%$, diagnóstico de leucemia linfocítica aguda, y el 10,2\% provenía del Departamento Médico de Abdomen. Las características demográficas y clínicas de los pacientes con fungemias distribuidos por etiología se describen en la Tabla 1 .

Comparando con las fungemias causadas por Candida no albicans, las causadas por C. albicans tuvieron mayor proporción en pacientes provenientes del Departamento de Abdomen $(17,2 \%$ frente a $8,0 \%)$. Cuando se analizaron estos pacientes y las especies de Candida, se encontró que C. albicans solo fue más frecuente que C. tropicalis (17,2\% frente a 5,2\%). 
Tabla 1. Características demográficas y clínicas de los pacientes con fungemias distribuidas según etiología (285 casos)

\begin{tabular}{|c|c|c|c|c|c|}
\hline \multirow{2}{*}{ Variables } & C. albicans & C. no albicans & Otras levaduras & Hongos filamentosos & Total \\
\hline & n (\%) & n (\%) & n (\%) & n (\%) & n (\%) \\
\hline \multicolumn{6}{|l|}{ Grupo etario (años) } \\
\hline $0-19$ & $13(20,3)$ & $52(27,8)$ & $11(40,8)$ & $2(28,6)$ & $78(27,4)$ \\
\hline $20-39$ & $12(18,8)$ & $47(25,1)$ & $5(18,5)$ & $2(28,6)$ & $66(23,1)$ \\
\hline $40-59$ & $20(31,2)$ & $49(26,2)$ & $5(18,5)$ & $2(28,6)$ & $76(26,7)$ \\
\hline 60 a más & $19(29,7)$ & $39(20,9)$ & $6(22,2)$ & $1(14,2)$ & $65(22,8)$ \\
\hline \multicolumn{6}{|l|}{ Sexo } \\
\hline Masculino & $30(46,9)$ & $80(42,8)$ & $16(59,3)$ & $4(57,1)$ & $130(45,6)$ \\
\hline Femenino & $34(53,1)$ & $107(57,2)$ & $11(40,7)$ & $3(42,9)$ & $155(54,4)$ \\
\hline \multicolumn{6}{|l|}{ Departamento Médico } \\
\hline Medicina & $27(42,2)$ & $120(64,2)$ & $14(51,9)$ & $5(71,4)$ & $166(58,3)$ \\
\hline Pediatría & $8(12,5)$ & $30(16,0)$ & $8(29,6)$ & $0(0,0)$ & $46(16,1)$ \\
\hline Abdomen & $11(17,2)$ & $15(8,0)$ & $3(11,1)$ & $0(0,0)$ & $29(10,2)$ \\
\hline Otros $^{a}$ & $18(28,1)$ & $22(11,8)$ & $2(7,4)$ & $2(28,6)$ & $44(15,4)$ \\
\hline \multicolumn{6}{|l|}{ Tipo de neoplasia } \\
\hline Tumor sólido & $34(54,0)$ & $59(31,6)$ & $4(14,8)$ & $3(42,9)$ & $100(35,1)$ \\
\hline Neoplasia hematológica & $29(46,0)$ & $128(68,4)$ & $23(85,2)$ & $4(57,1)$ & $184(64,6)$ \\
\hline \multicolumn{6}{|l|}{ Diagnóstico oncológico } \\
\hline Leucemia mieloide aguda & $5(7,8)$ & $32(17,1)$ & $4(14,8)$ & $1(14,3)$ & $42(14,8)$ \\
\hline Leucemia linfocítica aguda & $7(10,9)$ & $70(37,4)$ & $12(44,5)$ & $2(28,6)$ & $91(31,9)$ \\
\hline Linfoma no Hodgkin & $13(20,3)$ & $17(9,1)$ & $3(11,1)$ & $1(14,3)$ & $34(11,9)$ \\
\hline Tumor gastrointestinal & $14(21,9)$ & $31(16,6)$ & $2(7,4)$ & $1(14,3)$ & $48(16,9)$ \\
\hline Tumor genitourinario & $12(18,8)$ & $16(8,6)$ & $0(0,0)$ & $2(28,6)$ & $30(10,5)$ \\
\hline Otros $^{b}$ & $13(20,3)$ & $21(11,2)$ & $6(22,2)$ & $0(0,0)$ & $40(14,0)$ \\
\hline \multicolumn{6}{|l|}{ Neutropenia } \\
\hline No & $46(71,9)$ & $64(34,2)$ & $11(40,7)$ & $3(42,9)$ & $124(43,5)$ \\
\hline Sí & $18(28,1)$ & $123(65,8)$ & $16(59,3)$ & $4(57,1)$ & $161(56,5)$ \\
\hline
\end{tabular}

a Urología, Ginecología, Unidad de Cuidados Intensivos, Cabeza y Cuello, Neurocirugía, Tórax, Mamas y Tejidos, Ortopedia, Nefrología, Unidad de Terapia Intensiva.

b Leucemia mieloide crónica, leucemia linfocítica crónica, linfoma Hodgkin, mieloma múltiple, tumor de cabeza y cuello, tumor de pulmón, tumor de hígado, tumor de piel, osteosarcoma, histiocitosis de células Langerhans, leucemia aguda de células no especificadas, síndrome hemofagocitico, sin diagnóstico.

Las fungemias por C. albicans (54,0\%) fueron más frecuentes que las causadas por Candida no albicans $(31,6 \%)$ y otras levaduras $(14,8 \%)$ en pacientes con tumor sólido. Al analizar las neoplasias hematológicas y las especies de Candida se encontró que $C$. tropicalis fue más frecuente que C. albicans $(77,6 \%$ frente a $46,0 \%)$.

C. albicans fue más frecuentemente aislado que Candida no albicans en pacientes con linfoma no Hodgkin $(20,3 \%$ frente a $9,1 \%$ ) y tumor genitourinario $(18,8 \%$ frente a $8,6 \%)$. Cuando se analizaron el linfoma no Hodgkin y las especies de Candida, se encontró que C. albicans solo fue más frecuente que C. glabrata (20,3\% frente a 0,0\%). Mientras que, en tumor genitourinario, se encontró que C. albicans solo fue más frecuente que C. tropicalis (18,8\% frente a 6,7\%).

Por el contrario, hubo menor proporción de fungemias por C. albicans $(10,9 \%)$ en comparación con las causadas por Candida no albicans $(37,4 \%)$ y otras levaduras (44,5\%) en pacientes con leucemia linfocítica aguda (LLA). Cuando se analizaron la LLA y las especies de Candida, se encontró que $C$. albicans fue menos frecuente que C. tropicalis $(10,9 \%$ frente a $44,8 \%)$ y otras candidas (10,9\% frente a $35,3 \%)$.

Las fungemias por C. albicans $(71,9 \%)$ tuvieron mayor proporción que las causadas por Candida no albicans (34,2\%) y otras levaduras $(40,7 \%)$ en pacientes sin neutropenia. Cuando se analizaron estos pacientes y las especies de Candida, se encontró que $C$. albicans fue más frecuente que C. tropicalis $(71,9 \%$ frente a $26,1 \%)$ y otras candidas $(71,9 \%$ frente a $29,4 \%)$.

Además, al analizar el tipo de neoplasia y el estado de neutropenia se encontró que, en pacientes con tumores sólidos, el estado de no neutropenia fue más frecuente que el estado de neutropenia $(67,5 \%$ frente a $10,6 \%)$. 
Las candidemias (251 casos) representaron el 88,1\% de las fungemias, se aisló Cándida no albicans en el 65,6\% (187 casos), siendo C. tropicalis el más aislado con 47,0\% (134 casos). Otras levaduras se aislaron en el 9,5\% (27 casos) y los hongos filamentosos en el 2,5\% (7 casos) (Tabla 2).

Candida no albicans predominó durante los cinco años de revisión, aunque tuvo una disminución en su frecuencia, del 73,5\% en el 2012 al 60,6\% en el 2015. La frecuencia de $C$. albicans se incrementó de 14,7\% en 2012 a 31,0\% en el 2015. Otras levaduras y hongos filamentosos se mantuvieron por debajo del $14,0 \%$ y el 4,0\%, respectivamente (Figura 1).

Se encontró variación en la frecuencia de las fungemias y en el estado de neutropenia, en relación con el tiempo trascurrido. Las fungemias en pacientes sin neutropenia se incrementaron de $23,5 \%$ en el 2012 a 55,0\% en el 2016. Además, se analizó la variación en la frecuencia de las fungemias y en el tipo de neoplasia, y no se encontró un incremento sostenido en el tiempo.

Se observó variación en la frecuencia de las fungemias y el tipo de neoplasia, el diagnóstico oncológico, el estado de neutropenia y la etiología, en relación con la edad. Las fungemias en pacientes con tumores sólidos y con cáncer gastrointestinal se incrementaron con la edad, de $17,9 \%$ en el grupo etario de 0 a 19 años a $75,4 \%$ en el grupo etario de 60 años a más, y de 2,5\% en el grupo etario de 0 a 19 años a $50,8 \%$ en el grupo etario de 60 años a más, respectivamente. Similarmente, las fungemias en pacientes sin neutropenia

Tabla 2. Distribución de la etiología de las fungemias (285 casos)

\begin{tabular}{lcc}
\hline Agente etiológico & n & \% \\
\hline Levaduras & 64 & 22,5 \\
C. albicans & 134 & 47,0 \\
C. tropicalis & 18 & 6,3 \\
C. parapsilosis & 18 & 6,3 \\
C. glabrata & 4 & 1,4 \\
C. Krusei & 5 & 1,7 \\
C. guillermondi & 3 & 1,1 \\
C. lusitaniae & 5 & 1,7 \\
Otras Candidas ${ }^{\text {a }}$ & 6 & 2,1 \\
C. neoformans & 4 & 1,4 \\
Cryptococcus sp. & 6 & 2,1 \\
T. asahii & 4 & 1,4 \\
Trichosporon sp. & 4 & 1,4 \\
Rhodotorula spp. & 3 & 1,1 \\
Otras levaduras ${ }^{\text {b }}$ & & \\
Hongos filamentosos & 4 & 1,4 \\
Fusarium spp. & 3 & 1,1 \\
Acremonium spp. & & \\
\hline
\end{tabular}

${ }^{a}$ C. famata $(\mathrm{n}=1)$, C. kefyr $(\mathrm{n}=1)$, C. zeylanoides $(\mathrm{n}=1)$, Candida $\mathrm{sp} .(\mathrm{n}=2)$

${ }^{\mathrm{b}}$ Khodamoeba ohmeri $(\mathrm{n}=2)$, Geotrichum $\mathrm{spp} .(\mathrm{n}=1)$

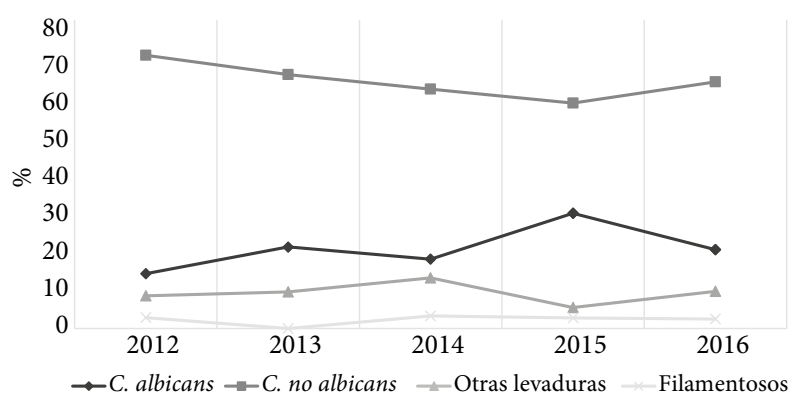

Figura 1. Distribución porcentual de la etiología agrupada de las fungemias, del 2012 al 2016

aumentaron de $34,6 \%$ en el grupo etario de 0 a 19 años a $81,5 \%$ en el grupo etario de 60 años a más. Finalmente, las fungemias por C. albicans aumentaron de $16,7 \%$ en el grupo etario de 0 a 19 años a $29,2 \%$ en el grupo etario de 60 años a más (Figura 2).

\section{DISCUSIÓN}

El tipo de neoplasia y el estado de neutropenia parecen estar relacionados con la etiología de las fungemias. El predominio de las fungemias por C. albicans en pacientes con tumores sólidos y el de las fungemias por Candida no albicans, (principalmente C. tropicalis en LLA) y otras levaduras en neoplasias hematológicas puede deberse al origen de la infección. En tumores sólidos el origen es principalmente endógeno, mientras que en neoplásias hematológicas el origen sería por levaduras no comensales ${ }^{(12,13)}$. En el mismo sentido, encontramos mayor frecuencia de C. albicans en tumores genitourinarios y de abdomen, ya que $C$. albicans es habitual comensal en esas regiones anatómicas y causante de complicaciones posoperatorias en cirugías abdominales ${ }^{(14,15)}$.

Por otro lado, la mayor frecuencia de fungemias por $C$. albicans en pacientes no neutropénicos y por $C$. tropicalis en pacientes neutropénicos puede explicarse por la relación entre el tipo de neoplasia y el estado de neutropenia. Las fun-

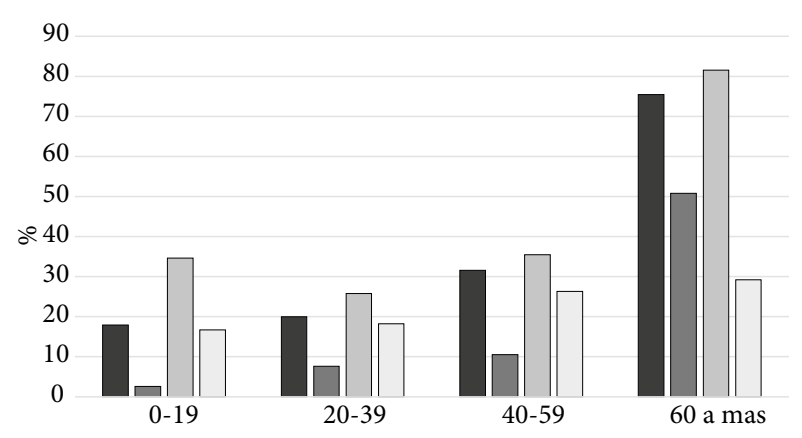

घTumor sólido $\square$ Tumor gastrointestinal $\square$ Sin Neutropenia $\square$ C. albicans

Figura 2. Proporción de fungemia según característica clínica y grupo etario 
gemias por C. albicans se presentan en tumores sólidos sin neutropenia, mientras que $C$. tropicalis se presenta en neoplasias hematológicas con neutropenia. Algunos estudios muestran que el estado de no neutropenia es más frecuente en pacientes con tumores sólidos que en neoplasias hematológicas ${ }^{(16,17)}$.

La diversidad en el tipo y frecuencia de la etiología de las fungemias determinan la terapia antifúngica adecuada. Las candidemias fueron las fungemias más frecuentes, con predominio de Candida no albicans siendo C. tropicalis el más aislado. La mayor frecuencia de Candida no albicans debido a C. tropicalis en la etiología de las fungemias ya se ha descrito en algunos estudios de nuestra región ${ }^{(18,19)}$. La posible asociación entre el cáncer y las fungemias por C. tropicalis puede explicar este predominio en la etiología ${ }^{(19)}$.

Se encontraron posibles tendencias y estas aparentan tener correlación clínica, pero precisan de mayor evaluación. El incremento en el tiempo de las fungemias en pacientes sin neutropenia, indicaría que la neutropenia es cada vez menos necesaria para el desarrollo de estas micosis invasivas. Este incremento no sería por un aumento de los tumores sólidos ya que estos permanecen invariables el tiempo. El incremento continuo de las fungemias según edad en pacientes con tumores sólidos (particularmente tumores gastrointestinales) puede deberse a características propias de estas neoplasias. Los pacientes con tumores sólidos tienen mayor edad que los que tienen neoplasias hematológicas ${ }^{(20)}$.

Finalmente, el continuo aumento de las fungemias en pacientes sin neutropenia y por C. albicans con el incremento de la edad pueden explicarse por la relación entre estas dos características y los tumores sólidos, algo ya descrito en párrafos anteriores. Los tumores sólidos se presentan con más frecuencia en personas de más edad y, a su vez, están relacionados con estados de no neutropenia y etiología por C. albicans.

\section{REFERENCIAS BIBLIOGRÁFICAS}

1. Paiva J, Pereira J, Tabah A, Mikstacki A, de Carvalho F, Koulenti D, et al. Characteristics and risk factors for 28-day mortality of hospital acquired fungemias in ICUs: data from the EUROBACT study. Critical Care. 2016; 20(1):53. doi: 10.1186/s13054-016-1229-1.

2. Cornely O, Gachot B, Akan H, Bassetti M, Uzun O, Kibbler C, et al. Epidemiology and outcome of fungemia in a cancer Cohort of the Infectious Diseases Group (IDG) of the European Organization for Research and Treatment of Cancer (EORTC 65031). Clin Infect Dis. 2015; 61(3):324331. doi: $10.1093 / \mathrm{cid} / \mathrm{civ} 293$.

3. Gaona-Flores V,Campos-Navarro L, Cervantes-Tovar R, Alcalá-Martínez E. The epidemiology of fungemia in an infectious diseases hospital in Mexico city: a 10-year retrospective review. Med Mycol. 2016; 54(6):600604. doi: $10.1093 / \mathrm{mmy} / \mathrm{myw} 017$.

4. Dong D, Li Z, Zhang L, Jiang C, Mao E, Wang X, et al. Clinical and microbiological investigation of fungemia from four hospitals in China. Mycopathologia. 2015; 179(5-6):407-414. doi: 10.1007/s11046-014-9855-0.

5. Castanheira M. Fungemia surveillance in Denmark demonstrates emergence of non-albicans Candida species and higher antifungal usage and
Nuestro estudio tuvo algunas limitaciones como la recopilación retrospectiva de los datos y la poca muestra para algunas categorías de las variables consideradas, lo cual impidió realizar un análisis inferencial. Asimismo, la identificación fúngica se realizó a nivel fenotípico, aunque las técnicas moleculares proveen una identificación más confiable, son costosas, complejas y poco aplicables a laboratorios hospitalarios.

En conclusión, nuestros hallazgos sugieren que las fungemias son causadas predominantemente por C. tropicalis, principalmente en pacientes con neoplasias hematológicas con neutropenia, mientras que las fungemias por C. albicans predominan en pacientes con tumores sólidos sin neutropenia. Además, conforme pasan los años se incrementan las fungemias en los pacientes sin neutropenia, y conforme aumenta la edad se incrementan las fungemias por C. albicans en los pacientes con tumores sólidos sin neutropenia.

Se recomienda realizar estudios que evalúen la sensibilidad antifúngica de los aislados, la asociación con factores de riesgo para la aparición y el desenlace de las fungemias. Esto permitirá entender el escenario actual de las fungemias en pacientes con cáncer y tomar medidas adecuadas de control y tratamiento.

Contribuciones de autoría: FV participó en el diseño del estudio, el establecimiento del protocolo de trabajo, el procesamiento y análisis de los resultados, y la escritura final del manuscrito. JV y KC participaron en el diseño del estudio, y en la recolección y procesamiento de datos. $\mathrm{EB}$ y SM participaron en el diseño del estudio y en la recolección de datos. SO e IC participaron en el diseño del estudio, la recolección de datos, y el procesamiento y análisis de los resultados. Todos los autores revisaron y aprobaron la versión final del manuscrito.

Fuentes de financiamiento: Autofinanciado.

Conflictos de interés: Los autores declaran no tener ningún conflicto de interés.

resistance rates than in other nations. J Clin Microbiol. 2018; 56(4):e0190717. doi: 10.1016/j.diagmicrobio.2016.02.009.

6. Capoor M, Gupta D, Verma P, Sachdeva H. Rare yeasts causing fungemia in immunocompromised and haematology patients: case series from Delhi. Indian J Med Microbiol. 2015; 33(4):576. doi: 10.1128/JCM.01907-17. doi: 10.4103/0255-0857.167320.

7. Castanheira M, Messer S, Rhomberg P, Pfaller M. Antifungal susceptibility patterns of a global collection of fungal isolates: results of the SENTRY Antifungal Surveillance Program (2013). Diagn Microbiol Infect Dis. 2016; 85(2):200-204. doi: 10.1016/j.diagmicrobio.2016.02.009.

8. Freydiere AM, Guinet R, Boiron P. Yeast identification in the clinical microbiology laboratory; phenotypical methods. Med Mycol. 2001; 39: 9-33. doi: 10.1080/mmy.39.1.9.33.

9. Larone DH, Larone DH. Medically important fungi: a guide to identification. New York: Elsevier; 1987.

10. Ascioglu S, Rex JH, De Pauw B, Bennett JE, Bille J, Crokaert F. et al. Defining opportunistic invasive fungal infections in immunocompromised 
patients with cancer and hematopoietic stem cell transplants: an international consensus. Clin Infect Dis. 2002;34(1):7-14. doi: 10.1086/323335.

11. Urabe, A. Clinical features of the neutropenic host: definitions and initial evaluation. Clin Infect Dis. 2004; 39(Supplement_1), S53-S55. doi: $10.1086 / 383055$.

12. Nucci M, Anaissie E. Revisiting the source of candidemia: skin or gut?. Clin Infect Dis. 2001; 33(12):1959-1967. doi: 10.1086/323759.

13. Bassetti M, Peghin M, Carnelutti A, Righi E, Merelli M, Ansaldi F. et al. Clinical characteristics and predictors of mortality in cirrhotic patients with candidemia and intra-abdominal candidiasis: a multicenter study. Intensive Care Med. 2017; 43(4):509-518. doi: 10.1007/s00134-017-4717-0.

14. Bassetti M, Righi E, Ansaldi F, Merelli M, Scarparo C, Antonelli M, et al. A multicenter multinational study of abdominal candidiasis: epidemiology, outcomes and predictors of mortality. Intensive Care Med. 2015; 41(9):1601-1610. doi: 10.1007/s00134-015-3866-2.

15. Bergamasco M, Garnica M, Colombo A, Nucci M. Epidemiology of candidemia in patients with hematologic malignancies and solid tumours in Brazil. Mycoses. 2013; 56(3):256-263. doi: 10.1111/myc.12013.
16. Gustinetti G, Mikulska M. Bloodstream infections in neutropenic cancer patients: a practical update. Virulence. 2016; 7(3):280-297. doi: 10.1080/21505594.2016.1156821.

17. Noskin G. Management of Infectious Complication in Cancer Patients. Boston, MA: Springer; 2007.

18. Colombo A, Nucci M, Park B, Nouér S, Arthington-Skaggs B, da Matta $\mathrm{D}$, et al. Epidemiology of candidemia in Brazil: a nationwide sentinel surveillance of candidemia in eleven medical centers. J Clin Microbiol. 2006; 44(8):2816-2823. doi: 10.1128/JCM.00773-06.

19. Tang H, Liu W, Lin H, Lai C. Epidemiology and prognostic factors of candidemia in cancer patients. PLoS One. 2014; 9(6):e99103. doi: 10.1371/ journal.pone.0099103.

20. Puig-Asensio M, Ruiz-Camps I, Fernández-Ruiz M, Aguado J, Muñoz $\mathrm{P}$, Valerio M, et al. Epidemiology and outcome of candidaemia in patients with oncological and haematological malignancies: results from a population-based surveillance in Spain. Clin Microbiol Infect. 2015; 21(5):491-e1. doi: 10.1016/j.cmi.2014.12.027. 